\title{
On subclasses of groups without free subsemigroups
}

\author{
B. Bajorska, O. Macedońska
}

August 17, 2013

\begin{abstract}
The paper is inspired by the question of A.Shalev about possible coincidence of the class of collapsing groups and groups satisfying positive laws. We split the class of collapsing groups for subclasses, corresponding to different functions on natural numbers and give a positive answer for some of them.
\end{abstract}

\section{Introduction}

Let $\mathcal{F}_{2}$ be a free semigroup generated by $x, y$ and let $u(x, y), v(x, y) \in \mathcal{F}_{2}$. By a (positive) law of degree $n$ we mean here a binary expression

$$
u(x, y)=v(x, y),
$$

where $x$ (and $y$ ) has the same exponent sum on both sides; the first (and the last) letters in $u$ and $v$ are different; the length of $u$ (equal to the length of $v$ ) is $n$. From now on by a pair of elements we mean an ordered one. We say that a pair $g, h$ of elements in a group satisfies a law (1) if the equality $u(g, h)=v(g, h)$ holds. A subset satisfies a law (1) if every pair of elements in the subset does.

Many authors considered properties of groups, which do not contain nonabelian free subsemigroups or, which is the same, groups with no free 2generator subsemigroups [1], [2], [3], [7], [9]. We call groups of such type $\left(\right.$ no $\left.\mathcal{F}_{2}\right)$-groups.

1991 AMS Subject Classification 20F19 
The notion of a collapsing group was introduced in [10] as $(n, m)$-collapsing. This definition is equivalent ([10], Lemma 2.2) to the following: a group $G$ is called collapsing if and only if there exists $n$, such that $G$ is $(n, 2)$-collapsing for some $n$, that is for any 2-element subset $A$ in $G$, the inequality $\left|A^{n}\right|<|A|^{n}$ holds.

Positive laws defining nilpotent groups were found in 1953 by Mal'cev [5]. Till 1996 [8] every known group satisfying positive laws was an extension of a nilpotent group by a group of a finite exponent. There still are many problems concerning positive laws.

We compare the above classes of groups. In $\left(\right.$ no $\left.\mathcal{F}_{2}\right)$-groups every pair of elements satisfies some law (1), while for groups with a positive law there exists a common law for all pairs. A collapsing group is $\left(n o \mathcal{F}_{2}\right)$-group with a restriction on degrees of the laws. So there are inclusions

$$
\left\{\begin{array}{c}
\left(\text { no } \mathcal{F}_{2}\right)- \\
\text { groups }
\end{array}\right\} \geq\left\{\begin{array}{c}
\text { collapsing } \\
\text { groups }
\end{array}\right\} \geq\left\{\begin{array}{c}
\text { positive } \\
\text { law }
\end{array}\right\} .
$$

In [11] A. Shalev posed the question: does every collapsing group satisfy a positive law?

A positive answer was found for residually finite groups ([11], Corollary C) and for finitely generated soluble groups ([10], Theorem 4.2). We can extend the second result to all soluble groups.

Theorem 1 Every soluble collapsing group $G$ satisfies a positive law.

Proof Let $G$ be a $(n, 2)$-collapsing group, then $G$ is a $\left(n o \mathcal{F}_{2}\right)$-group. Let $H$ denote any finitely generated subgroup in $G$. Obviously $H$ is also $(n, 2)$ collapsing. By Theorems 4.7, 4.12 [9], every finitely generated soluble $\left(\right.$ no $\left.\mathcal{F}_{2}\right)$ group is nilpotent-by-finite and hence residually finite. So $H$ is residually finite. It follows from Theorem B [11], that every finitely generated residually finite $(n, 2)$-collapsing group satisfies some positive law $M_{c, i}$, where $c, i$ depends on $n$ only. Thus we conclude that every finitely generated subgroup in $G$ satisfies the same law $M_{c, i}$, and hence $G$ satisfies the positive law, as required.

Since, in general, the problem may have a negative answer we suggest another approach to the question. We show that a group $G$ is collapsing if and only if for all $m$, every $m$-element subset $A_{m}$ in $G$ satisfies a positive law of degree $\leq \varphi(m)$, where $\varphi(m)$ depends on $m$ only (Theorem 2 ). So we 
obtain a refinement of the problem: For which $\varphi(m)$ : if every $m$-element subset in $G$ satisfies a positive law of degree $\varphi(m)$, then $G$ has to satisfy a positive law?

To simplify futher studies we need the following criterion for a group to be collapsing.

Lemma 1 A group $G$ is collapsing if and only if there exists $k$ such that every 2-element subset in $G$ satisfies a law of degree $\leq k$.

Proof Let every pair of elements in $G$ satisfy a law of degree $\leq k$. If $A=$ $\{g, h\}$ and a pair $g, h$ satisfies a law $s(x, y)=t(x, y)$ of degree $d \leq k$, then in the set $A^{k}$ two products coincide: $g^{k-d} s(g, h)=g^{k-d} t(g, h)$. So we have $\left|A^{k}\right|<|A|^{k}$ which implies that $G$ is collapsing.

If a group $G$ is collapsing then it is $(n, 2)$-collapsing for some $n$ and for any subset $A=\{g, h\}$ the inequality $\left|A^{n}\right|<|A|^{n}$ holds. Then at least two products in $A^{n}$ coincide, that is $s(g, h)=t(g, h)$, say. So we have that every pair $g, h$ of elements in $G$ satisfies a law of a length $n$, which implies a balanced law $s(g, h) t(g, h)=t(g, h) s(g, h)$ of degree $k \leq 2 n$ (after cancellation).

\section{Notations:}

- Let $m$ be a fixed natural.

$\left(A_{m} \in \mathcal{L}\right)$-group is a group in which every $m$-element subset satisfies some law (1) (depending on the subset). We write $\left(A_{m} \in \mathcal{L}_{d}\right)$ if the length of these laws is bounded by $d$.

- $(A \in \mathcal{L})$-group is a group which is $\left(A_{m} \in \mathcal{L}\right)$-group for every $m$. We write $\left(A \in \mathcal{L}_{d}\right)$ if the length of all laws is bounded by $d$.

- Let $\varphi=\varphi(m)$ be a natural function of $m$.

$\left(A \in \mathcal{L}_{\varphi}\right)$-group is a group $G$ such that for every $m, G$ is $\left(A_{m} \in \mathcal{L}_{\varphi(m)}\right)$ group. If the function $\varphi$ is bounded we write $\left(A \in \mathcal{L}_{\text {bound }}\right)$

It is clear that the class of $\left(A \in \mathcal{L}_{d}\right)$-groups contains all groups which satisfy a positive law. The class of $(A \in \mathcal{L})$-groups contains all groups which locally satisfy positive laws.

We show that collapsing groups are $\left(A \in \mathcal{L}_{\varphi}\right)$-groups.

For every $\left(A \in \mathcal{L}_{\varphi}\right)$-group $G$ we introduce the following minimal function

Definition $1 f(m)$ is the minimal number such that every m-element subset in $G$ satisfies a positive law of degree $\leq f(m)$. 
So every $\left(A \in \mathcal{L}_{\varphi}\right)$-group is $\left(A \in \mathcal{L}_{f}\right)$-group.

Theorem $2 A$ group $G$ is collapsing if and only if it is $\left(A \in \mathcal{L}_{f}\right)$-group, where $\forall m, f(m) \leq[f(2)]^{m^{2}-m}$.

Proof By Lemma 1 there exists $k$ such that every 2-element subset in $G$ satisfies a law of degree $\leq k$, so every pair of elements also does. We assume $k$ minimal with this property. So in terms of the function $f, k=f(2)$. We show that any $m$-element subset $A_{m}$ in $G$ satisfies a law of degree $\leq k^{m^{2}-m}$. Since every reflexive pair $(a, a)$ satisfies any balanced law (1), we need to find a common law only for $m^{2}-m$ pairs in $A_{m}$. These pairs we put in some order $a, b ; c, d ; e, f ; \ldots$ There exists a law $u_{1}(x, y)=v_{1}(x, y)$ of degree $\leq k$ such that $u_{1}(a, b)=v_{1}(a, b)$. We assume that $u_{1}(c, d)$ and $v_{1}(c, d)$ are different. However there exists a law $u_{2}(x, y)=v_{2}(x, y)$ of degree $\leq k$, such that: $u_{2}\left(u_{1}(c, d), v_{1}(c, d)\right)=v_{2}\left(u_{1}(c, d), v_{1}(c, d)\right)$. Since the law $u_{2}(x, y)=v_{2}(x, y)$ is balanced, it is also satisfied by the pair of equal elements $u_{1}(a, b), v_{1}(a, b)$. So the common law for two pairs $a, b$ and $c, d$ is $u_{2}\left(u_{1}(x, y), v_{1}(x, y)\right)=v_{2}\left(u_{1}(x, y), v_{1}(x, y)\right)$, of degree $\leq k^{2}$.

Assume that for $i-1$ pairs in $A_{m}$ a common law $a(x, y)=b(x, y)$ of degree $\leq k^{i-1}$ is found and that the $i$-th pair $g, h$ does not satisfy it. We consider elements $a(g, h)$ and $b(g, h)$. There exists a law $u_{3}(x, y)=v_{3}(x, y)$ of degree $\leq k$ such that $u_{3}(a(g, h), b(g, h))=v_{3}(a(g, h), b(g, h))$. Then the common law for all $i$ pairs is $u_{3}(a(x, y), b(x, y))=v_{3}(a(x, y), b(x, y))$ of degree $\leq k^{i}$. By adding new pairs and repeating the step we find the law for $A_{m}$ of degree $\leq k^{m^{2}-m}=[f(2)]^{m^{2}-m}$. So $f(m) \leq[f(2)]^{m^{2}-m}$ and the group is $\left(A \in \mathcal{L}_{f}\right)$-group, as required.

Conversely, in $\left(A \in \mathcal{L}_{f}\right)$-group every 2-element subset satisfies a law of degree $\leq f(2)$ and by Lemma $1, G$ is collapsing.

Theorem 3 A group $G$ satisfies a law of degree $\leq d$ if and only if $G$ is a $\left(A_{2 D} \in \mathcal{L}_{d}\right)$-group, where $D$ is the number of laws of degrees $\leq d$.

We assume that the laws are ordered. Let $\mathcal{P}_{i} \in G \times G$ be a subset of pairs which do not satisfy the $i$-th law of degree $\leq d$. If $G$ does not satisfy a positive law, then no $\mathcal{P}_{i}$ is empty. We take a pair from every $\mathcal{P}_{i}$ and get a subset of $2 D$ elements which does not satisfy a law of degree $\leq d$. Hence $G$ is not a $\left(A_{2 D} \in \mathcal{L}_{d}\right)$-group, which is a contradiction. The converse is obvious.

Corollary $1 G$ satisfies a law if and only if $G$ is $\left(A \in \mathcal{L}_{\text {bound }}\right)$-group. 
Proof The first implication is clear. Conversely, let $f(m) \leq d, m \in N$, for some fixed $d$. Then $f(2 D) \leq d$, where $D$ is the number of laws of degree $\leq d$. So $G$ is $\left(A_{2 D} \in \mathcal{L}_{d}\right)$-group and hence by Theorem $3, G$ satisfies a law.

Theorem 4 The chain of classes (2) coincides with

$$
\left\{\begin{array}{c}
(A \in \mathcal{L})- \\
\text { groups }
\end{array}\right\}>\left\{\begin{array}{c}
\left(A \in \mathcal{L}_{f(m)}\right)- \\
\text { groups }
\end{array}\right\} \geq\left\{\begin{array}{c}
\left(A \in \mathcal{L}_{\text {bound }}\right)- \\
\text { groups }
\end{array}\right\},
$$

or more precisely

$$
\left\{\begin{array}{c}
\left(\forall m, A_{m} \in \mathcal{L}\right)- \\
\text { groups }
\end{array}\right\}>\left\{\begin{array}{c}
\left(\forall m, A_{m} \in \mathcal{L}_{f(m)}\right)- \\
\text { groups }
\end{array}\right\} \geq\left\{\begin{array}{c}
\left(\exists d, \forall m, A_{m} \in \mathcal{L}_{d}\right)- \\
\text { groups }
\end{array}\right\} .
$$

Proof In $\left(\right.$ no $\left.\mathcal{F}_{2}\right)$-group every pair of elements satisfies some law. It is shown in the proof of Theorem 2, that for a finite number of such pairs $a_{i}, b_{i}$ there exists a common law, so $G$ is a $\left(n o \mathcal{F}_{2}\right)$-group if and only if for every $m$ it is an $\left(A_{m} \in \mathcal{L}\right)$-group. Because of Theorem 2 and Corollary 1 we need to see only that the first inequality is strict. We consider an infinite direct product $\Pi$ of free nilpotent groups of increasing nilpotency classes. Since $\Pi$ is locally nilpotent then by [5], $\Pi$ locally satisfies positive laws and so for every $m, \Pi$ is an $\left(A_{m} \in \mathcal{L}\right)$-group.

If assume that $\prod$ is $\left(A \in \mathcal{L}_{f}\right)$-group then, as a consequence, every pair of elements satisfies some law of degree $\leq f(2)$. These laws form a finite subset $\left\{u_{i}(x, y)=v_{i}(x, y), i=1,2, \ldots, D\right\}$. So as in [11] we conclude that $\Pi$ has to satisfy the commutator law

$$
\left[\left[\ldots\left[\left[u_{1} v_{1}^{-1}, u_{2} v_{2}^{-1}\right], u_{3} v_{3}^{-1}\right], \ldots\right], u_{D} v_{D}^{-1}\right]=1 .
$$

Since $\prod$ does not satisfy any law, we have a contradiction and hence $\prod$ is not $\left(A \in \mathcal{L}_{f}\right)$-group, which finishes the proof.

Remark The above law does not imply a positive law, because it is satisfied in a free metabelian group which does not satisfy a positive law [5].

\section{When $\left(A \in \mathcal{L}_{f}\right)$-group satisfies a positive law}

We can give the following properties of the function $f$, defined in the previous section. 
Lemma 2 (i) $f$ is not a decreasing function, (ii) if $m \geq n$ and $M$ are some fixed naturals, then every $\left(A_{m} \in \mathcal{L}_{M}\right)$-group is $\left(A_{n} \in \mathcal{L}_{M}\right)$-group. (iii) if for a group $G, \forall m \geq 16, f(m) \leq \log _{4} m$, then $G$ satisfies a law of degree $\leq \log _{4} m$.

Proof Properties (i) and (ii) follow from the definition. To prove (iii) we denote $d=\log _{4} m$ (for $m<16$ it does not make any sense) then the condition $f(m) \leq \log _{4} m$ gives $f\left(4^{d}\right) \leq d$ and hence by property (ii), $G$ is $\left(A_{4^{d}} \in \mathcal{L}_{d}\right)$ group. We use a rough calculation to get $D$ (the number of laws of degree $\leq d$. The number of all words of the length $d$ is $2^{d}$ and the number of laws of degree $\leq d$ is $D \leq 2^{d}\left(2^{d}-1\right) / 2<2^{2 d-1}$. So $2 D<4^{d}$. By property (ii) $\left(A_{4^{d}} \in \mathcal{L}_{d}\right)$-group is $\left(A_{2 D} \in \mathcal{L}_{d}\right)$-group and hence, by Theorem $3, G$ satisfies a law of degree $\leq d=\log _{4} m$ as required.

Remark The above calculation is too rough not only because we obtained many times (after cancellation) the same law, but also: (1) the fact that pairs $a, b$ and $b, a$ are always in the same $A_{m}$, implies that the laws $u(x, y)=$ $v(x, y)$ and $u(y, x)=v(y, x)$ are simultaneously satisfied and hence one can be removed, (ii) some law can be a consequence of another for the same pair $g, h \in G$, for example a pair of commuting elements satisfies any balanced law.

In [4] there was introduced a definition of a minimal set of laws. For a given $d$ we denote by $\mathcal{M}(d)$ a minimal set of laws of degree $\leq d$ such that if a subset $A$ satisfies a law of degree $\leq d$, then it satisfies a law in $\mathcal{M}(d)$. Now if denote $\mu(d)=2|\mathcal{M}(d)|$ it follows from the proof of Theorem 3

Corollary 2 Every $\left(A_{\mu(d)} \in \mathcal{L}_{d}\right)$-group satisfies a law of degree $\leq d$.

It is clear that if one of the four laws

$$
u(x, y)=v(x, y), v(x, y)=u(x, y), u(y, x)=v(y, x), v(y, x)=u(y, x)
$$

is satisfied in some subset, then the other three are also satisfied and hence only one belongs to $\mathcal{M}(d)$. We say that a law $u(x, y)=v(x, y)$ of degree $n$ is of the type $X^{k} Y^{l}$ if the first letter in $u$ is $x$, the exponent sum of $x^{\text {'s }}$ is $k$ and the exponent sum of $y$ 's is $l, k+l=n$. The law of the type $X^{k} Y^{l}$ is called standard if $k \leq l$ and $u(x, y)$ is lexicographically less than, or equal to $v(y, x)$.

So the set $\mathcal{M}(d)$ consists of standard laws. For example $\mathcal{M}(2)$ consists of one abelian law. If a set satisfies an abelian law then ii satisfies any other law )1). So $\mathcal{M}(3)$ also contains only one law $x y^{2}=y^{2} x$ and we get 
Corollary $3\left(A_{2} \in \mathcal{L}_{2}\right)$-group satisfies a positive law of degree 2. $\left(A_{2} \in \mathcal{L}_{3}\right)$ group satisfies a positive law of degree $\leq 3$.

Since both $x y=y x$ and $x y^{2}=y^{2} x$ imply $x^{2} y^{2}=y^{2} x^{2}$, we get

$\mathcal{M}(4) \subseteq\left\{(x y)^{2}=(y x)^{2},(x y)^{2}=y^{2} x^{2}, x y^{3}=y^{3} x, x y^{2} x=y x^{2} y, x^{2} y^{2}=y^{2} x^{2}\right\}$

So $|\mathcal{M}(4)| \leq 5$, and then by Corollary 2 we get: if every 10-element subset in a group satisfies some law of degree $\leq 4$, then the group satisfies a law of degree $\leq 4$ and we can write it as

Corollary $4\left(A_{10} \in \mathcal{L}_{4}\right)$-group satisfies a positive law of degree $\leq 4$.

Moreover by [4], such a group has to satisfy one of the following three laws:

$$
x y^{3}=y^{3} x, x y^{2} x=y x^{2} y, x^{2} y^{2}=y^{2} x^{2},
$$

which are minimal (as consequences) in the poset of laws of degree $\leq 4$.

As it is shown in [4], there are 13 standard laws of degree 5. Since the law $x y^{3}=y^{3} x$ implies $x^{2} y^{3}=y^{3} x^{2}$, we have $|\mathcal{M}(5)| \leq|\mathcal{M}(4)|+13-1=17$.

Corollary 5 Every $\left(A_{34} \in \mathcal{L}_{5}\right)$-group satisfies a positive law of degree $\leq 5$.

Using Lemma 2 and Corollaries 3,4,5 we prove

Theorem 5 If there exists at least one $m$ such that the point $(m, f(m))$ is in the area $\Omega$ (see figure 1 below) then $\left(A \in \mathcal{L}_{f}\right)$-group has a positive law.

Proof The construction is as follows. The horizontal axe $m$ denotes the number of elements in the set. The vertical axe $k$ denotes degree of the law. We mark the point $(M, K)$ if every $M$-element subset satisfies a law of degree $\leq K$. The point $(M, K)$ is called $\mathbf{P L}$ if when $f(M) \leq K$ then $\left(A \in \mathcal{L}_{f}\right)$-group has positive law. We give the list of PL points:

1. By corollaries $3,4,5,(2,2),(2,3),(10,4),(34,5)$ are PL.

2. By (iii) in Lemma 2 for $M \geq 16,2 \leq K \leq \log _{4} M,(M, K)$ are PL.

3. By (ii) in Lemma 2 if $(M, K)$ is PL then $(M+S, K)$ is PL. So from the above it follows that every point which is "on the right" of the PL point is PL itself. 


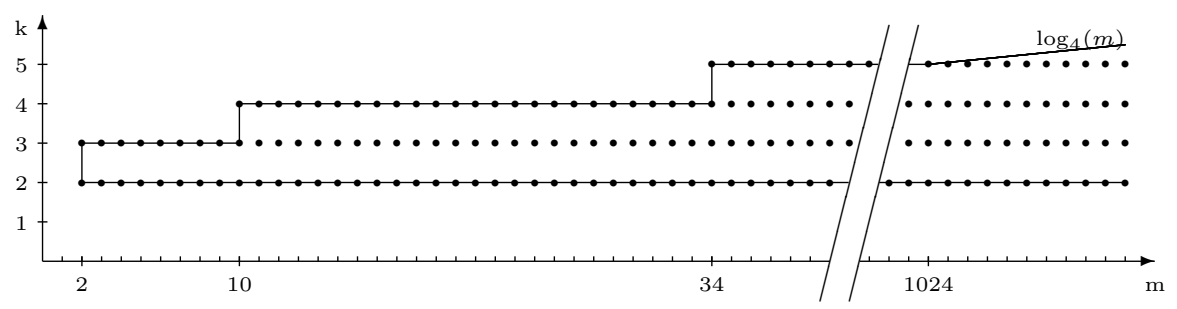

Figure 1: $\Omega$ area

We construct an area $\Omega$, which consist of PL points. Note that we consider only points with integer coordinates. It follows from the definition that if at least one point $(m, f(m)), m \in N$ belongs to $\Omega$ then $\left(A \in \mathcal{L}_{f}\right)$-group has to satisfy a positive law, as required.

Remark For $k=5$ from 1 . and 2. in Theorem 5 we get different minimal $m$, for which the point $(m, k)$ belongs to $\Omega$, namely 34 and 1024, respectively. So the latter one gives us much worse result. However for $k \geq 6$ the only we have is $m \geq 4096$. The result can be improved if we know the number of elements in $\mathcal{M}(d)$ for $d>5$, so it seems to be an open area for futher studies.

\section{References}

[1] G. Freiman, On two and three-element subsets of groups, Aeq. Math. 22, (1981), 140-152.

[2] M. Herzog, P. Longobardi, M. May, On a combinatorial problem in group theory, Israel Journal of Math. 82, (1993), 329-340.

[3] P. Longobardi, M. Maj, A. H. Rhemtulla, Groups with no free subsemigroups, Trans. Am. Math. Soc. 347, (1995), 1419-1427.

[4] O. Macedońska, M. Żabka, On equivalence of semigroup identities Matematica Scandinavica, to appear.

[5] A.I.Mal'cev, Nilpotent semigroups, Uchen. Zap. Ivanovsk. Ped. Inst. 4 (1953), 107-111. 
[6] B. H. Neumann, T. Taylor, Subsemigroups of nilpotent groups, Proc. Roy. Soc. Ser. A 274 (1963), 1-4.

[7] J. Okniński, "Semigroups of matrices", World Scientific, Singapore 1998.

[8] A.Yu.Ol'shanskii, A.Storozhev, A group variety defined by a semigroup law, J. Austral. Math. Soc. (Series A), 60, (1996), 255-259.

[9] J.M.Rosenblatt, Invariant measures and growth conditions, Trans. Am. Math. Soc. 193, (1974), 33-53.

[10] J.F.Semple, A.Shalev, Combinatorial conditions in residually finite groups, I, J.Algebra 157 (1993), 43-50.

[11] A.Shalev, Combinatorial conditions in residually finite groups, II, J.Algebra 157 (1993), 51-62.

Institute of Mathematics,

Silesian Technical University,

ul. Kaszubska 23,

44-100 Gliwice, Poland

e-mail: olga@zeus.polsl.gliwice.pl

e-mail: bajorska@zeus.polsl.gliwice.pl 